\title{
Prediction Method of Railway Freight Volume Based on Improved Neural
}

\author{
Network \\ Zhida Guo ${ }^{1,}$, Jingyuan $\mathrm{Fu}^{1, b}$ \\ ${ }^{1}$ School of Economics and Management, Dalian Jiaotong University, Dalian 116028 \\ ${ }^{a}$ email, ${ }^{b}$ email
}

Key words: Railway Freight Volume; Forecasting; General Regression Neural Network; Genetic Algorithm

\begin{abstract}
Railway freight transportation is an important part of national economy. Accurate forecast of railway freight volume is significant to the planning, construction, operation and decision-making of railways. After analyzing the application status of general regression neural network (GRNN) in prediction method of railway freight volume, this paper improves the performance of this model by using improved neural network. In the improved method, genetic algorithm (GA) is adopted to search the optimal spread which is the only factor of GRNN, and then the optimal spread is used for forecasting in GRNN. Finally, the railway freight volumes in the example are forecasted based on this method.
\end{abstract}

\section{Introduction}

Freight transportation is one of the core businesses in railways and this method of transportation plays an important role in the conveying of goods throughout the country. The national railway freight center was set up in 2013 which is facing new development opportunities at present.

To anticipate the future demand for railway freight volume is particularly urgent, and therefore accurate forecasts should be predicted in advance by using exist things and new resources.

Up to now, there are many available methods in the prediction of railway freight volume, including traditional forecasting methods and numerous modern intelligent algorithms. American scholars Brockweil and Davis (1991) analyzed and compared several commonly used time series models and found that the autoregressive integrated moving average model could successfully predict the effect of traffic flow [1].

Babcock (1999) [2] established time series model which is used to forecast the number of railway grain loading.

Godfrey and [3] (2000) Powell established an exponential smoothing model to forecast the daily demand for cargo transport.

For the domestic scholars, Li Hongqi (2003) [4] and Liu Kai (2004) [5] have demonstrated the feasibility fractal theory and rough set theory for China's railway freight volume forecast, and have proposed two methods to enhance this forecasting process.

Zhao Chuang (2004) et al. [6,7] established the railway freight volume forecast, generalized regression neural network model and the support vector machine model respectively.

And Liu Zhijie (2006) et al [8] used the radial basis function neural network for freight volume analysis and forecast.

Based on the analysis of the influence factors of freight volume, Geng Liyan (2012) et al. proposed the 
least square support vector machine model based on grey correlation analysis.

Guo Yuhua (2010) et al. [9] proposed the concept of the economic cycle stage parameter, thus established the Elman neural network forecast model based on the economic cycle.

Therefore, in this paper, based on the generalized regression neural network forecasting method, we introduce the genetic algorithm to improve research with the hope that the improved algorithm can provide a new approach for the railway freight volume forecasting, railway freight reform in our country, the railway planning and construction. It also provides the reference for the establishment of related policies.

\section{Performance Analysis of Railway Freight Volume Forecasting with Generalized Regression Neural Network}

Regression Neural Network General(GRNN) was proposed by Dr. Specht in 1991.

The theoretical advantage of GRNN as a predictive tool is that it does not need to be specified in advance, because the method uses the complex relationships provided by the examples to learn.

GRNN is based on nonlinear regression, the implementation of Parzen non-parametric estimation is based on the principle of maximum probability of computing network output. GRNN was used as it possesses a strong learning ability, good nonlinear approximation performance, robustness and the ability to be fault tolerant.

GRNN has these characteristics and advantages, compared to other methods. It is particularly suitable for railway freight volume forecast.

Training samples are taken in order to ascertain the GRNN, network structure and the neurons between the connection weights with the input. The GRNN needed to be adjusted as the parameters indicate only a smooth sigma factor, with large computational advantage. However, the prediction results have a great influence in determining to determine the improper smoothing factor.

Specht (1991) [10] had proposed a method called Multiple test methods to determine $\sigma$. Let the smooth factor in a range set up ahead of the ( $\sigma \mathrm{min}, \sigma \mathrm{max})$ in arithmetic changes.

Then, remove a few or individual training samples, and use the remaining samples to train the neural network.

Next sigma arithmetic was performed to remove minor or individual samples. This was to predict value and between sample values. Owing to a square error in the evaluation index, a sigma error minimum value was used as the optimal smoothing factor, for the final generalized regression neural network predictive.

Another way is the selection of smoothness factor, i.e. Conducting optimizing through function. Seng (2002) etc proposed to seek for optimum value through gradient descent. But when using this method, local extremum often cause the failure in optimizing.

\section{Improved Method of Forecasting Process}

The proposed improved method of the prediction process is divided into two parts. The first part: uses genetic algorithm to find the only optimum value of GRNN parameter smooth factors. Genetic algorithm is the main subject. The input is parameter values of genetic algorithm. This produces a random generation of smooth factor groups. The output is the optimum value of smooth factors.

The second part: involves conducting a GRNN prediction on optimal values of smooth factors. GRNN is the main subject. The input is index system data relevant to forecast and optimal smooth factor. The 
output is prediction results.

The first part is to achieve an improvement of genetic algorithm on GRNN. It is designed based on the genetic progress general regression neural network model. The basic procedure of the improved prediction method is shown in Figure.1.

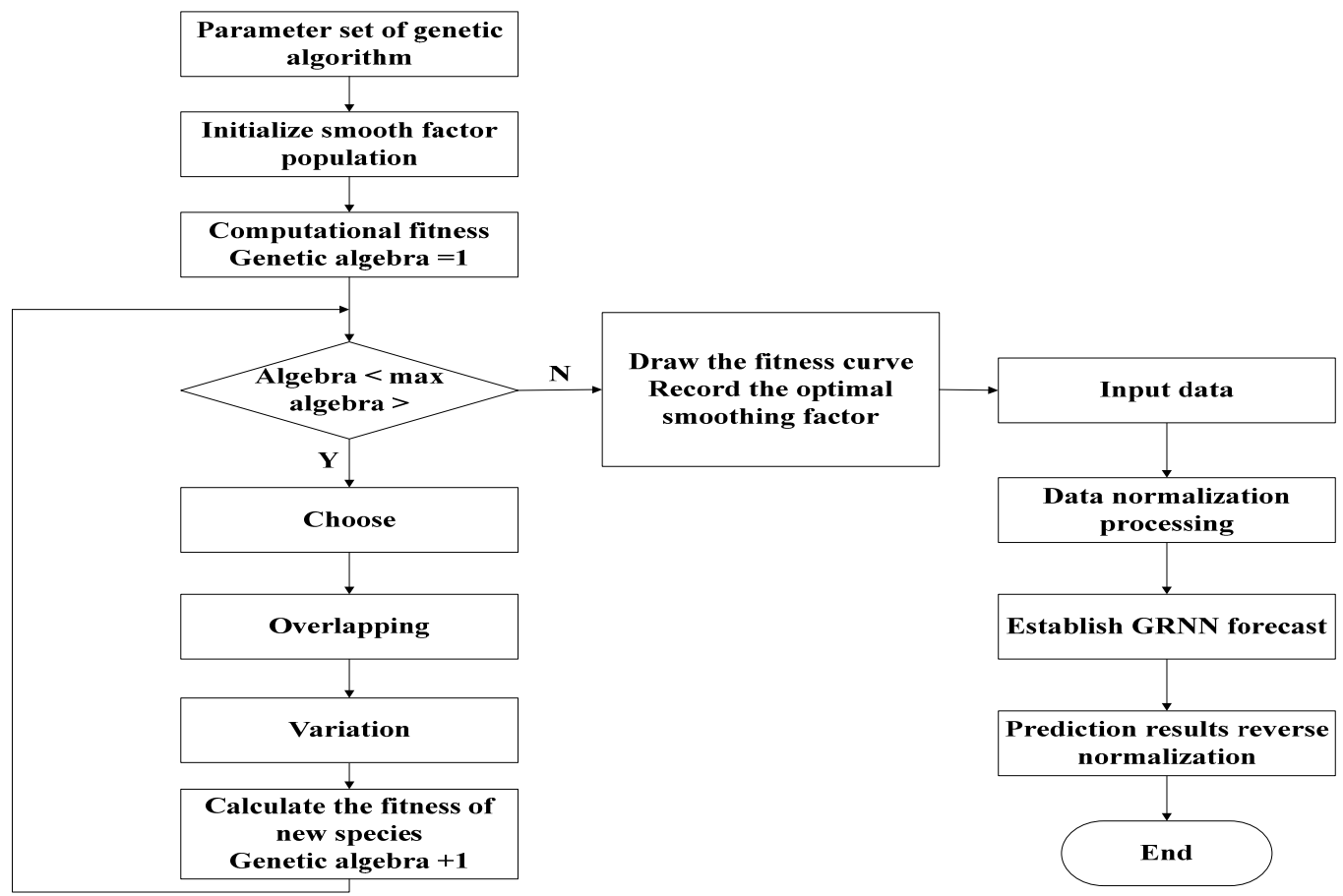

Figure 1 :genetic improvement generalized regression neural network method forecast flow chart

How to construct the fitness function is one of the most notable problems in genetic algorithm. In this paper, GRNN is established through fitness function. The adjacent five samples are selected and monitored. Target values of three samples adjacent to the previous five samples are predicted. The reciprocal of the euclidean distance between prediction results and true value is used here as fitness function.

The smaller the value of euclidean distance, the larger values form the fitness value. $\mathrm{X}$ is the true value, and $\mathrm{x}$ is the prediction value. Fitness value is:

$$
f(x)=\frac{1}{\sqrt{\sum_{i=1}^{3}\left(x_{i}-X_{i}\right)^{2}}}
$$

\section{Forecast Example}

In this paper, a new method of genetic improvement based on the generalized regression neural network is proposed, and is applied to railway freight volume forecasting.

But in general situation, it will take some time to obtain statistical data of these 11 influence factors.

This would impact promptness of predicting railway freight volumes, thus reducing the significance of prediction results.

The solution is to consider real-time supervision in order to obtain statistical data of relevant influencing factors. Other suitable prediction methods can also adopted to predict the values of 11 influence factors. Moreover, prediction values of these influencing factors can be regarded as input value, and conduct prediction of railway freight volumes. 
As most influence factors of railway freight volumes indicate stability, few changes or certain obvious tendency, such as gross domestic product. Based on development of the national economy, and macroeconomic regulation and control, increases are achieved between $6 \% \sim 8 \%$. So the method of exponential smoothing can be used to predict the 11 influence factors

Then substitute the prediction value of influence factors into the improved algorithm, in order to obtain the forecast value of railway freight volumes.

Such way of prediction__ data obtained through traditional prediction methods before using the improved algorithm_ utilized them to obtain the final prediction result they want, which are also of scientificity.

Because while using influence factors to predict railway freight volumes, influence factors have nonlinear relations with target values. Moreover, it is in conformity with mathematical model set by traditional prediction method.

As for the prediction of influence factors, based on the above analysis, it is suitable for traditional prediction method.

In other words, as for the prediction of different target values, different suitable prediction methods should be adopted to fit different target values.

Based on the above analysis, forecast is conducted on railway freight volumes in 2015 and 2016.

Considering that Chinese economic growth has entered the new normal state, and the overall international economic development has slowed down, while using SPSS software to predict influence factors, the method of exponential smoothing is adopted. Prediction values of railway freight volume influence factors from 2014 to 2016 is shown in Table 1.

\begin{tabular}{cccccccccccc}
\hline $\begin{array}{c}\text { Ye } \\
\text { ar }\end{array}$ & $X_{1}$ & $X_{2}$ & $X_{3}$ & $X_{4}$ & $X_{5}$ & $X_{6}$ & $X_{7}$ & $X_{8}$ & $X_{9}$ & $X_{10}$ & $X_{11}$ \\
\hline 201 & 61966 & 22552 & 25989 & 27334 & 2122 & 6071 & 5049 & 7403 & 10. & 48. & 9.2 \\
4 & 6.9 & 5.2 & 2.8 & 8.6 & 6.9 & 7.3 & 0.1 & 16 & 78 & 7 & 9 \\
201 & 67048 & 24036 & 26245 & 29167 & 2152 & 6124 & 5290 & 7651 & 11. & 49. & 9.1 \\
5 & 5.1 & 0.2 & 5 & 4.5 & 6.7 & 0.6 & 8.9 & 39 & 25 & 7 & 3 \\
201 & 72130 & 25519 & 26475 & 30998 & 2182 & 6176 & 5519 & 7899 & 11. & 50. & 9.0 \\
6 & 0 & 4.9 & 6.3 & 2 & 6.4 & 3.5 & 5.4 & 63 & 71 & 6 & 1 \\
\hline
\end{tabular}

Table 1: Influence Factors of railway freight volume in 2014 2016

In 2015 and 2016, the smooth factor fitness curve of the railway freight increment is predicted as shown in figure 3 and figure 4.
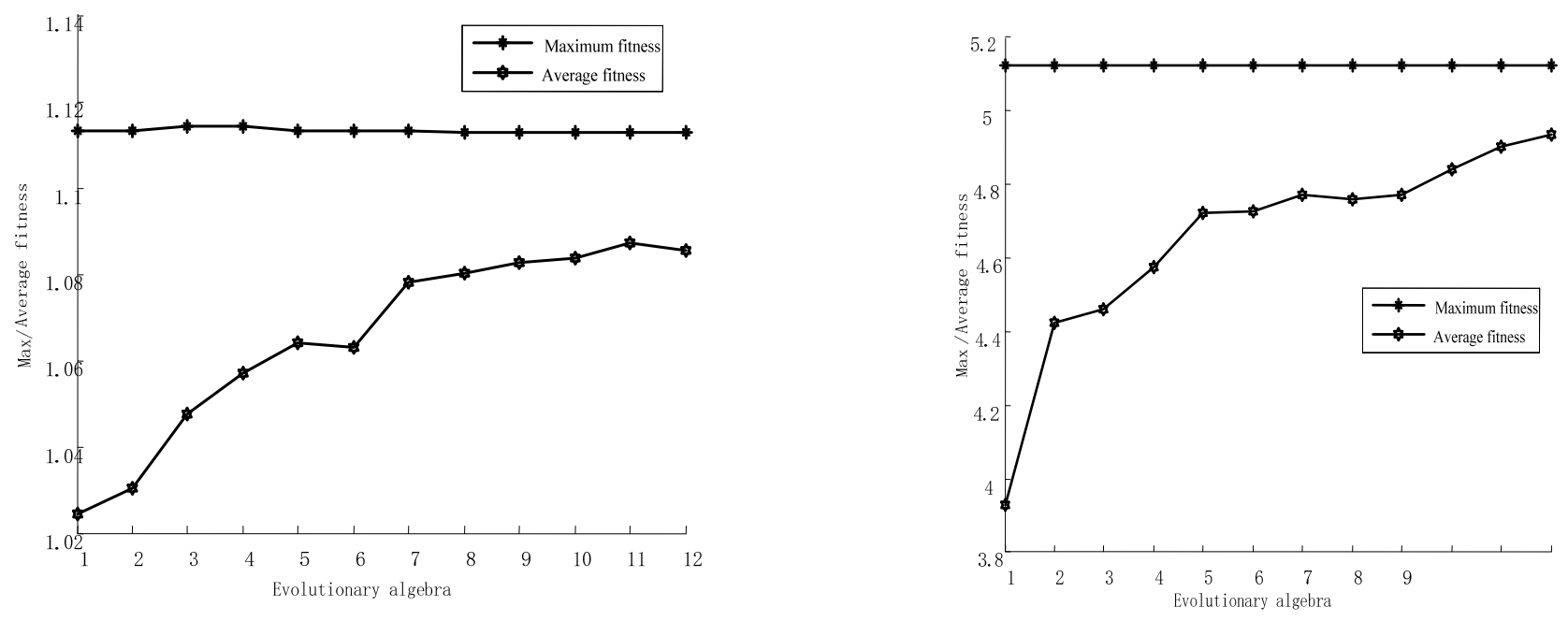
Figure2: 2015 Smoothing Factor of Railway Freight Figure3: 2016 SmoothingFactor of Railway Incremental Fitness Curves Prediction

Freight Incremental Fitness Curves Prediction

\section{Conclusion}

In this paper, a new method of genetic improvement based on the generalized regression neural network is proposed, and it is applied to railway freight volume forecasting.

Compared to the former method, genetic algorithm is more highly effective because it takes into consideration the subjective factors.

Prediction results show that using the same number of training samples, and forecasting the same target, genetic improvement of GRNN model prediction accuracy is better in the prediction of target values and therefore indicates a large improvement in forecast accuracy.

The set of improved algorithm to calculate fitness function, form an innovative method by using a ' $5+$ 3 ' mode, namely to establish a generalized regression neural network, with the continuous five training samples, capable of predicting the next 5 samples. The predicted results and the real value of the reciprocal of Euclidean distance as fitness function, ensure a smooth factor optimization which can be used to forecast any change in trend of the target data.

\section{Acknowledgements}

Fund Project: the National Natural Science Foundation of China (71271070 / G0107).

\section{Reference:}

[1] Brockweil P J, Davis R A.Time Series: Theory and Methods[M].New York: Springer-Verlag, 1991

[2] Michael W Babcock, Xiaohua Lu, Jerry Norton. Time Series Forecasting of Quarterly Railroad Grain Car Loadings[J]. Transportation Research Part E, 1999, 35:43-57.

[3] Gregory A Godfrey, Warren B Powell. Adaptive Estimation of Daily Demands with Complex Calendar Effects for Freight Transportation[J]. Transportation Research Part B,2000,34:451-469.

[4]LI Hong-qi, LIU Kai.Analysis of Railway Freight Volume Based on Fractal Theory[J].Journal of the China Railway Society,2003,25(3):19-23.

[5]LI Hong-qi, LIU Kai.Prediction of Railway Freight Volumes Based on Rough Set Theory[J].Journal of the China Railway Society,2004,26(3):1-7.

[6] ZHAO Chuang, LIU Kai, LI Dian-sheng. Freight Volume Forecast Based on GRNN[J].Journal of the China Railway Society,2004,26(1):12-15.

[7] ZHAO Chuang, LIU Kai, LI Dian-sheng. A study of Use Support Vector Machine Theory of Prediction Freight Volume[J]. Journal of the China Railway Society,2004,26(4):10-14.

[8] LIU Zhi-jie, JI Ling, YE Yu-ling, Etc.Study on Prediction of Railway Freight Volumes Based on RBF Neural Network[J].Journal of the China Railway Society,2006,28(5):1-5.

[9] GUO Yu-hua,CHEN Zhi-ya,FENG Fen-ling,etal.Railway Freight Volume Forecasting of Neural Network Based on Economic Cycles[J].Journal of the China Railway Society,2010,32(5):1-6.

[10] Donald F Specht. A General Regression Neural Network [J].IEEE Transactions on Neural Networks, $1991,2(6): 568-576$. 